\title{
Vibration Analysis of Cavitation in Kaplan Water Turbines
}

\author{
Sulo Lahdelma* Esko K. Juuso** \\ * Mechatronics and Machine Diagnostics Laboratory, Department of \\ Mechanical Engineering P.O.Box 4200, FI-90014 University of Oulu, \\ Finland, (email: sulo.lahdelma@oulu.fi) \\ ** Control Engineering Laboratory, Department of Process and \\ Environmental Engineering P.O.Box 4300, FI-90014 University of \\ Oulu, Finland, (email: esko.juuso@oulu.fi)
}

\begin{abstract}
Cavitation is harmful to water turbines and may cause operation delays of several weeks. The real-time detection of cavitation risk is increasingly important, and even narrow cavitation-free power ranges can be utilised in load optimisation. Higher derivative signals $x^{(3)}$ and $x^{(4)}$ calculated from acceleration signals are very suitable for detecting impacts. This paper introduces a generalised moment ${ }_{\sigma}^{\tau} M_{\alpha}^{p}$ which is defined by three parameters: the sensitivity of the moment improves when the order $p$ of the moment increases, especially when short sample time $\tau$ is used. In this study, sufficently good results were obtained with moments where the order of derivation $\alpha=4, p \approx 4$, and $\tau=3 \mathrm{~s}$. These moments detect the normal operating conditions, which are free of cavitation, and also provide a clear indication for cavitation risk at an early stage. Sufficiently long signals are required for producing reliable maximum moments and data for analysing short-term cavitation. On-line cavitation monitoring is feasible with this approach since the analysis does not need high frequency ranges and the sample times are very short. The moment can be analysed first, and it is then possible to obtain the cavitation index if the moment value exceeds the threshold. Data compression is very efficient as the detailed analysis only requires the feature values of the appropriate samples.
\end{abstract}

Keywords: cavitation; vibration analysis; higher order derivatives; feature extraction; water turbine.

\section{INTRODUCTION}

Condition monitoring provides a reliable, economical method for performing maintenance operations in modern industrial plants. The increasing number of measurement points and more complex problems require automatic fault detection. Advanced signal processing methods expose failures earlier and provide information on suitable operating conditions. Intelligent methods have increasingly been used in model-based fault diagnosis and intelligent analysers. The methods provide various techniques for combining a large number of features.

Cavitation is possible when vapour bubbles are formed in a liquid at a constant temperature. If pressure decreases below the saturated vapour pressure of the liquid at the same time, the bubbles grow. If this phenomenon takes place in a flow, the vapour bubbles grow intensively in a region of lower pressure. When the bubbles move to a higher pressure region, they collapse rapidly. The collapse takes place in a very short time period and causes high vibration levels, see [Brennen, 1995]. Traditionally, there have been efforts to detect cavitation using vibration, pressure, acoustic emission or sound measurements. A variety of studies concentrate on the cavitation of pumps, see [Cudina, 2003], [Alfayez et al., 2005] and [Al-Hashmi et al., 2005].
Cavitation is extremely harmful to turbines, as it damages the surfaces of runners and flow channels, e.g. a revision of the runner may cause delays of several weeks in the operation of the turbine. Cavitation and avoiding cavitation in water turbines has been investigated in some recent studies: [Roussopoulos and Monkewitz, 2000], [Bahaj and Myers, 2003], and [Escaler et al., 2006].

The power ranges should be selected in a way that minimises the possibility of cavitation. For instance, if one wants to produce as high output powers as possible at flood periods, advantages and disadvantages can be estimated when the severity of cavitation is known at the maximum power levels. As water turbines are used for fast reaction on fluctuations in electricity consumption, the power need to be changed quickly. Research on the hydropower plant modelling and control focus on turbine control with large load variation in the power system, see [Kishor et al., 2007].

The real-time detection of cavitation risk is increasingly important in both low and high power ranges. Even narrow cavitation-free power ranges can be utilised in load optimisation. Cavitation in water turbines has often been examined with standard $v_{r m s}$ measurements in the frequency range $10-1000 \mathrm{~Hz}$ or by selecting $1 \mathrm{~Hz}$ as the lower cut-off frequency [Juuso and Lahdelma, 2006]. However, practical experiences have shown that this analysis does not provide a sufficient picture of cavitation in the case of Kaplan tur- 


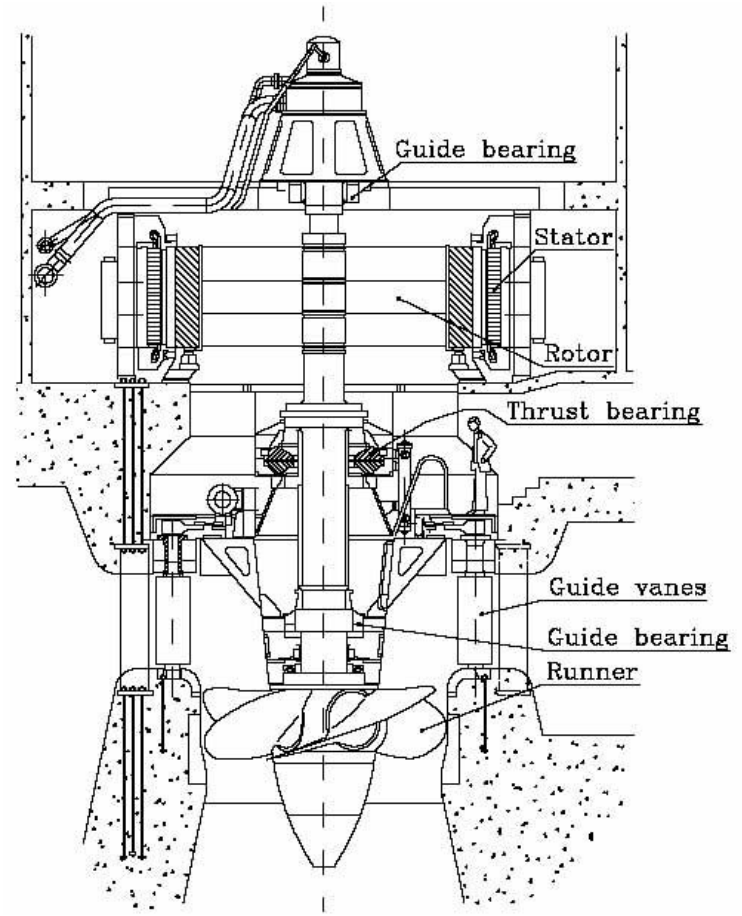

Fig. 1. Kaplan water turbine.

bines (Fig. 1). Higher order time derivatives have already been used to improve this analysis, see [Lahdelma et al., 1999].

The measurement signals traditionally used in vibration analysis are displacement, velocity and acceleration, i.e. $x^{(0)}, x^{(1)}$, and $x^{(2)}$. The signals $x^{(3)}$ and $x^{(4)}$ are very suitable for condition monitoring when the ability to detect impacts is important, see [Lahdelma, 1995] and [Lahdelma et al., 1999]. Lahdelma has used time derivatives of a higher order than acceleration, such as $x^{(3)}$ and $x^{(4)}$, in various condition-monitoring studies and features of these signals are also very sensitive in detecting cavitation in water turbines as seen in references [Lahdelma et al., 1999] and [Lahdelma, 2002].

Intelligent methods provide various techniques for combining a large number of features, see [Juuso, 1994] and [Juuso, 1999]. Linguistic equations introduced in [Juuso and Leiviskä, 1992] are designed for integrating knowledge and data in the development of nonlinear multivariable systems for intelligent process analysis, process control, fault diagnosis and forecasting, see [Juuso, 2004]. An insight to the process operation is maintained since all the modules can be assessed on the basis of expert knowledge, and membership definitions relate the measurements to appropriate linguistic terms on different operating areas.

Juuso and Lahdelma [2006] introduced a nonlinear scaling approach to features generated from vibration measurements and compared several cavitation indices constructed from these features in a water turbine.

This paper continues the cavitation analysis by comparing different features and sample times. A new generalised moment is introduced and compared to the previously studied features in model-based condition monitoring.

\section{VIBRATION MEASUREMENTS}

The turbine shown in Figure 1 has sleeve bearings and four blades and its rotation speed is $115 \mathrm{rpm}$. The turbine operates with a constant rotation speed and the output power is controlled by varying the volume flow rate of water through the turbine. Acceleration measurements were carried out vertically on the supporting bearings at 29 power levels varying from 1.5 to $59.4 \mathrm{MW}$ [Juuso and Lahdelma, 2006, Juuso et al., 2007, Lahdelma et al., 2007].

A Wilcoxon accelerometer model 726 with a permanent magnet was used in the measurements and signals were recorded using a Casio DA-7 16-bit DAT recorder in the frequency range $10 \mathrm{~Hz}$ to $20 \mathrm{kHz}$. The recorded acceleration signals were transferred to a computer using the LabVIEW 8.0 software and NI PCI-4472 24-bit data acquisition card. The sampling frequency in the data transfer was $12800 \mathrm{~Hz}$, which is rather low but sufficient in this case. The use of a higher sampling frequency would naturally increase the absolute values of different features but also increase sample file size.

An analogue differentiator/integrator was used in [Juuso and Lahdelma, 2006]. The analogue signal was differentiated and integrated by means of analogue differentiator/integrator MIP 1518ID2 manufactured by MIP Оy. The linear range of the equipment was from 2 to 2000 $\mathrm{Hz}$, and the upper limit was defined by means of a low pass filter. Sharp band-pass filtering was performed for the velocity signal and its frequency range was from 100 to $1000 \mathrm{~Hz}$. The signals $x^{(3)}$ and $x^{(4)}$ were obtained in frequency range 10 to $2000 \mathrm{~Hz}$. The lower limit $10 \mathrm{~Hz}$ is based ISO standard of vibration measurements in condition monitoring.

Certain features at varying frequency ranges were calculated from the stored acceleration signals using LabVIEW 8.0 software. The features were processed using the MATLAB software, version 7.3. The numerical derivation and integration of the acceleration signals were performed with LabVIEW, and all the signals were filtered by means of a sixth order Butterworth bandpass filter. The frequency ranges were $10-1000 \mathrm{~Hz}, 10-2000 \mathrm{~Hz}, 10-3000 \mathrm{~Hz}$ and $10-4000 \mathrm{~Hz}$. In this way, the time domain signals were generated at different power levels, see [Juuso et al., 2007].

Acceleration signals can reveal cavitation, but $x^{(4)}$ is more sensitive [Juuso et al., 2007]. The $x^{(4)}$ signal at the $2 \mathrm{MW}$ shows strong, stochastic impacts. For instance, the signal at $10 \mathrm{MW}$ has slightly more impacts, whereas the level of the impacts is lower. The signal levels at the cavitation-free power range are very low. Vibration levels grow when the power is increased above the clearly cavitation-free area, but the detected impacts are significantly weaker than in the 2 and $10 \mathrm{MW}$ measurements.

\section{FEATURE EXTRACTION}

In a previous study, strong cavitation was observed at power levels 2, 10 and 59.4 MW. There is a favourable, cavitation-free operating range from 13 to $40 \mathrm{MW}$. For the low power range, i.e. cases of 1.5-1.8, 3-6, 8, 9, 11 and $12 \mathrm{MW}$, these short periods of cavitation were seen and heard from the acceleration signals as there are fairly 


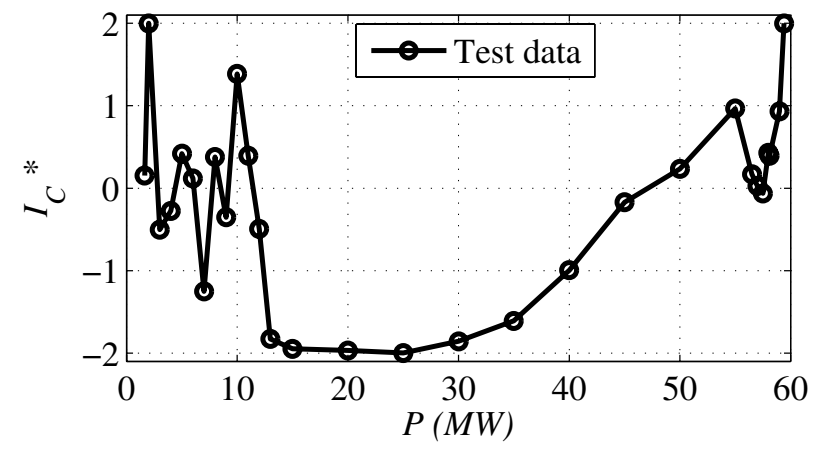

Fig. 2. Knowledge-based cavitation index.

strong impacts in the turbine. The impacts were much weaker than in the stronger cavitation cases and clearly stronger than in the case of $7 \mathrm{MW}$. For the high power range, i.e. cases of $45,50,56.5,57$ and $57.5 \mathrm{MW}$, the cavitation periods are seen and heard as a slightly higher noise level. The cases 58 and 58.1 MW have a continuously higher noise level, see [Juuso and Lahdelma, 2006].

The cavitation indices based on the signals $x^{(3)}$ and $x^{(4)}$ efficiently indicated all the levels of cavitation. If all the index values $I_{C}^{(4)}$ above $40 \mathrm{MW}$ are slightly reduced, also the cavitation index supports the fact that the favourable operating range extends to $50 \mathrm{MW}$. The index value for strong cavitation points at the low power range is emphasised by increasing the index values $I_{C}^{(4)}$ for the low power range. The resulting knowledge-based cavitation index $I_{C}^{*}$ is presented in Figure 2. The indices $I_{C}^{(1)}$ based on the features of velocity do not indicate cavitation, see [Juuso and Lahdelma, 2006].

Signals, $x^{(2)}, x^{(3)}$, and $x^{(4)}$ have been analysed in each frequency range for rms values, kurtosis and peak values, see [Juuso et al., 2007]. The kurtosis of each signal correlates with the index $I_{C}^{*}$ in the low power range: the strong cavitation at $2 \mathrm{MW}$ is clearly detected. For the power range from $13 \mathrm{MW}$, kurtosis is close to value 3, which corresponds to a Gaussian signal, i.e. kurtosis does not give an indication of cavitation in the high power range. The spikes caused by cavitation are hidden in the signal since the noise level is increasing. However, the indication is achieved with the rms values, which includes the effects of spikes and noise. Both the features need to be combined in the power range $3 \ldots 12 \mathrm{MW}$ : the cavitation point at 10 MW corresponds to high values in both the features. Only short periods of cavitation at $5 \mathrm{MW}$ are needed to raise kurtosis when the signal levels are low, i.e. the rms value is low. An alternative feature for kurtosis is peak value, which has fairly similar changes in the low power range and small changes in the high power range. [Juuso et al., 2007]

Widening the frequency range makes the features more sensitive in detecting cavitation at 10 and 59.4 MW. Also the absolute values of the features increase with the widening frequency range. The changes in kurtosis and peak values are different in different power ranges, e.g. the relative height of the spike becomes stronger at 10 MW and weaker at $5 \mathrm{MW}$. However, expanding the upper limit frequency from 2000 to $4000 \mathrm{~Hz}$ does not improve possibilities to observe cavitation at the power level of 2 MW. Thus the detection of cavitation does not require the use of very high upper limit frequencies. Even the upper limit frequency of $1000 \mathrm{~Hz}$ seems to be sufficient for detecting strong cavitation in this turbine. Model-based cavitation indices are needed for a more detailed analysis. [Juuso et al., 2007]

Previous studies have shown that several features need to be combined in cavitation analysis. To compare the possibilities of different approaches, a generalised moment is introduced here:

$$
{ }_{\sigma}^{\tau} M_{\alpha}^{p}=\frac{1}{N\left(\sigma_{\alpha}\right)^{p}} \sum_{i=1}^{N}\left|x_{i}^{(\alpha)}-\bar{x}^{(\alpha)}\right|^{p},
$$

where the real number $\alpha$ is the order of derivation, the real number $p$ is the order of the moment, $\tau$ is the sample time $(s)$, and $\bar{x}^{(\alpha)}$ and $\sigma_{\alpha}$ the mean and the standard deviation, respectively, calculated for the signal $x^{(\alpha)}$. The number of signal values $N=\tau N_{s}$, where $N_{s}$ is the number of samples per second. The order of derivation ranges from 1 corresponding to velocity to 4 , which corresponds to the signal $x^{(4)}$. The moment ${ }_{\sigma}^{\tau} M_{\alpha}^{2}=1$, and the moment ${ }_{\sigma}^{\tau} M_{\alpha}^{4}$ correspond to the kurtosis of the signal $x^{(\alpha)}$.

The moments were studied in the power range 1.5 . .59.4 MW from the measurements collected with sampling frequency $12800 \mathrm{~Hz}$. The order $p$ was in the range from 0.2 to 8 , and totally 11 different sample times were used: $\tau=1,2, \ldots, 6,8,10,20,30$, and 40 seconds. The length of the signals $x^{(1)}, x^{(3)}$, and $x^{(4)}$ was 50 seconds.

In the cavitation-free power range, the signal $x^{(4)}$ is close to a Gaussian distribution as can be seen from the moment ${ }_{\sigma}^{\tau} M_{\alpha}^{p}$ which is close to 3 when the order $p=4$. The sample time $\tau$ does not have any practical effect in this range. In cavitation cases, the order $p$ has a very strong effect if $p>2$. Therefore, the other power levels were studied by comparing them to the case at $15 \mathrm{MW}$.

The maximum values of the moments ${ }_{\sigma}^{\tau} M_{4}^{p}$, which were calculated for different sample times, are compared in low and high power levels, e.g. for the sample time $\tau=3 \mathrm{~s}$ the maximum is obtained from 16 samples, each with 38400 signal values. The values in Figure 3 are relative values compared to the cavitation-free case at $15 \mathrm{MW}$. The strong cavitation at $2 \mathrm{MW}$ and $10 \mathrm{MW}$ is clearly detected with all $\tau$ values if the $p$ value is close to 4 or higher (Figs. 3(a) and $3(\mathrm{~b}))$. The fairly good areas between the cavitation cases have values close to the cavitation-free cases. High values are also obtained at 5 and $8 \mathrm{MW}$ which has not as strong cavitation as at 2 and $10 \mathrm{MW}$. The moments become smaller with increasing sample time $\tau$ (Fig. 3(b)). However, the moments calculated at $5 \mathrm{MW}$ decrease less than the others.

The cavitation case at $59.4 \mathrm{MW}$ is only detected by the moments based on short signals (Fig. 3(c)). For long sample times, the moments become much smaller (Fig. $3(\mathrm{~d}))$. The levels of the signal $x^{(4)}$ are quite high at the high power range and considerable impacts take place only seldom. Most of the impacts are hidden. In the cavitationfree power ranges, the moment value can occasionally be even lower than in the comparison case at $15 \mathrm{MW}$. 


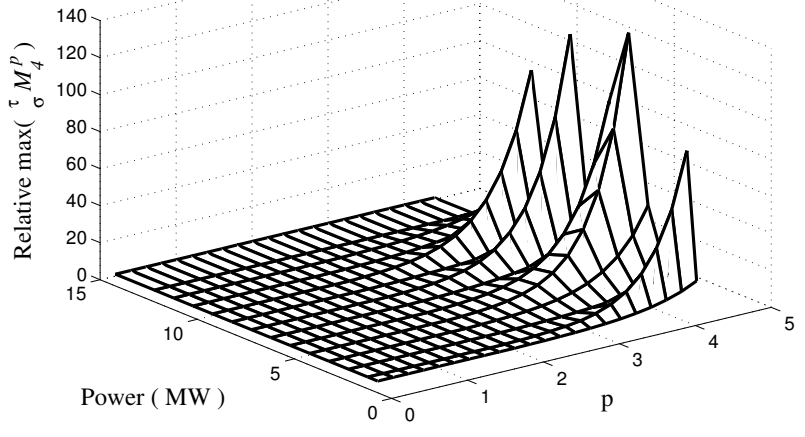

(a) Relative $\max \left({ }_{\sigma}^{\tau} M_{4}^{p}\right)$ in the low power range when $\tau=3 s$.

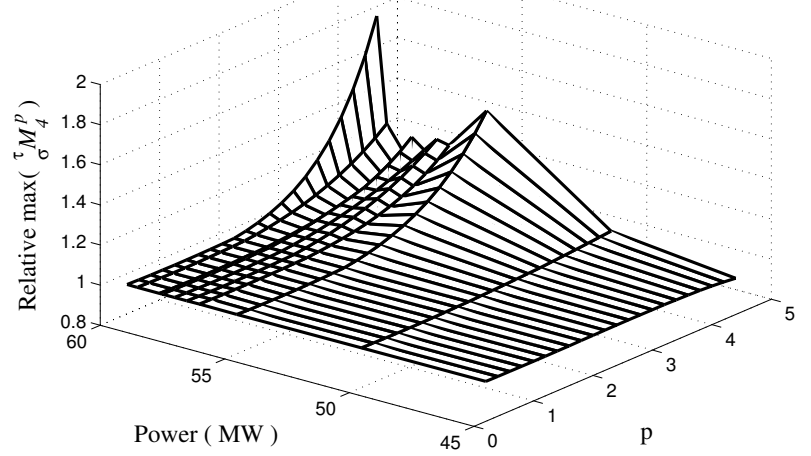

(c) Relative $\max \left({ }_{\sigma}^{\tau} M_{4}^{p}\right)$ in the high power range when $\tau=3 s$.

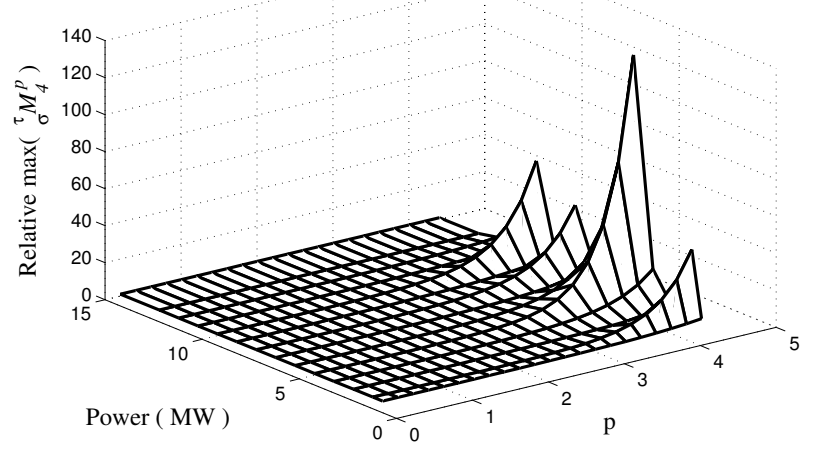

(b) Relative $\max \left({ }_{\sigma}^{\tau} M_{4}^{p}\right)$ in the low power range when $\tau=40$ s.

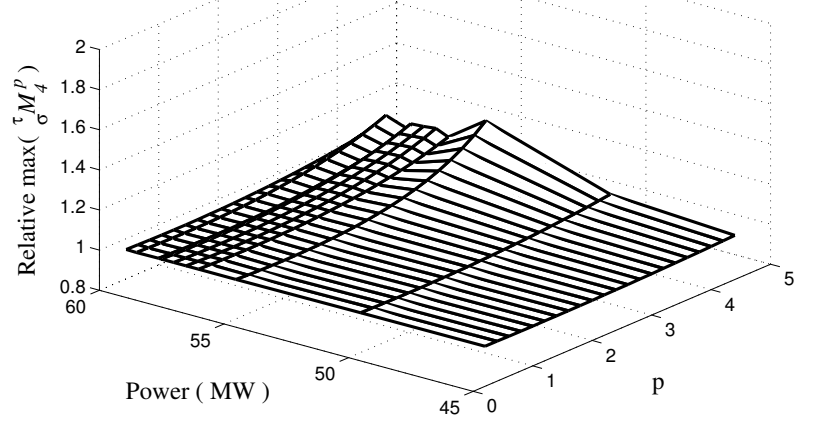

(d) Relative $\max \left({ }_{\sigma}^{\tau} M_{4}^{p}\right)$ in the high power range when $\tau=40 s$.

Fig. 3. Relative maximum moment ${ }_{\sigma}^{\tau} M_{4}^{p}$ for the signal $x^{(4)}$ in the frequency range $10-2000 \mathrm{~Hz}$.

The moments ${ }_{\sigma}^{\tau} M_{4}^{4}$ calculated for the sample time 40 seconds correspond to the kurtosis values calculated for the signal $x^{(4)}$ in [Juuso et al., 2007] and [Lahdelma et al., 2007]. The other moments in Figure 4 are kurtosis values calculated for shorter sample times: 1, 2 an 3 seconds. In the low power range, the most sensitive results are obtained with the sample time 3 seconds (Fig. 4). The cavitation-free cases are correctly classified by the moment values close to 3 . The cavitation case at 59.4 MW is also detected with the short sample times.

The moments ${ }_{\sigma}^{\tau} M_{1}^{4}$ calculated for shorter sample times show some improvements but the results are much poorer than with the moments ${ }_{\sigma}^{\tau} M_{4}^{4}$. The cavitation case at 10 MW is clearly detected, and the moment values at $2 \mathrm{MW}$ are also slightly higher than the kurtosis of the long sample time in these cases (Fig. 5) but the operation at the power ranges around $5 \mathrm{MW}$ is even poorer.

The generalised moment ${ }_{\sigma}^{\tau} M_{\alpha}^{p}$ can be defined by means of three parameters $\alpha, p$, and $\tau$. Quite short sample times $\tau$ can be used in the cavitation analysis. The moments ${ }_{\sigma}^{\tau} M_{4}^{p}$ calculated for shorter sample times and $p$ values around 4 provide useful features and an alternative for the kurtosis based on long sample times. Short sample times also provide new possibilities for analysing shortterm cavitation periods.

\section{MODELLING}

The moments indicate possible cavitation but one moment value is not enough for a detailed analysis. The moments should be combined with other features, i.e. rms values used in [Juuso et al., 2007] are needed to get the correct sequence for the severity of cavitation in the low power range. This model is closely related to the model used in [Juuso and Lahdelma, 2006] where peak values were calculated in a different way. Also the fraction of the values exceeding the normal range has clear similarities with the rms values.

Features are transformed to a linguistic scale from -2 to 2 through nonlinear scaling for the linguistic equation (LE) models in the same way as in the previous studies [Juuso, 2004, Juuso and Lahdelma, 2006, Juuso et al., 2007]. The scaling can be performed for the features in different frequency ranges by means of membership definitions. As the shape of the functions is quite similar, the features provide a good basis for a model-based analysis.

The detailed model-based analysis is excluded from this paper. Models for the cavitation index can be developed in different frequency ranges analysed from the features obtained at ten power levels, as presented in [Juuso et al., 2007]. The training set has to contain examples of cavita- 

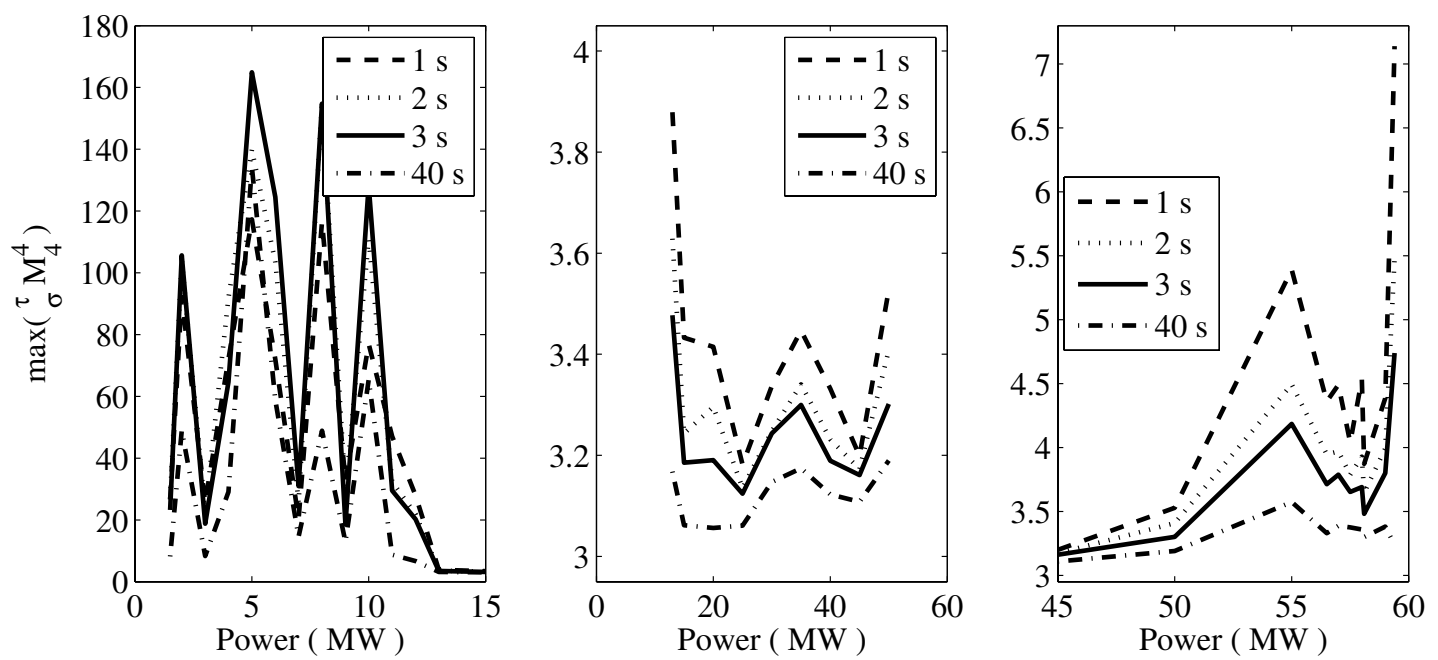

Fig. 4. Maximum moment of the signal $x^{(4)}$ calculated using different sample lengths for three power ranges in the frequency range $10-2000 \mathrm{~Hz}$. The maximum values are obtained from several samples.
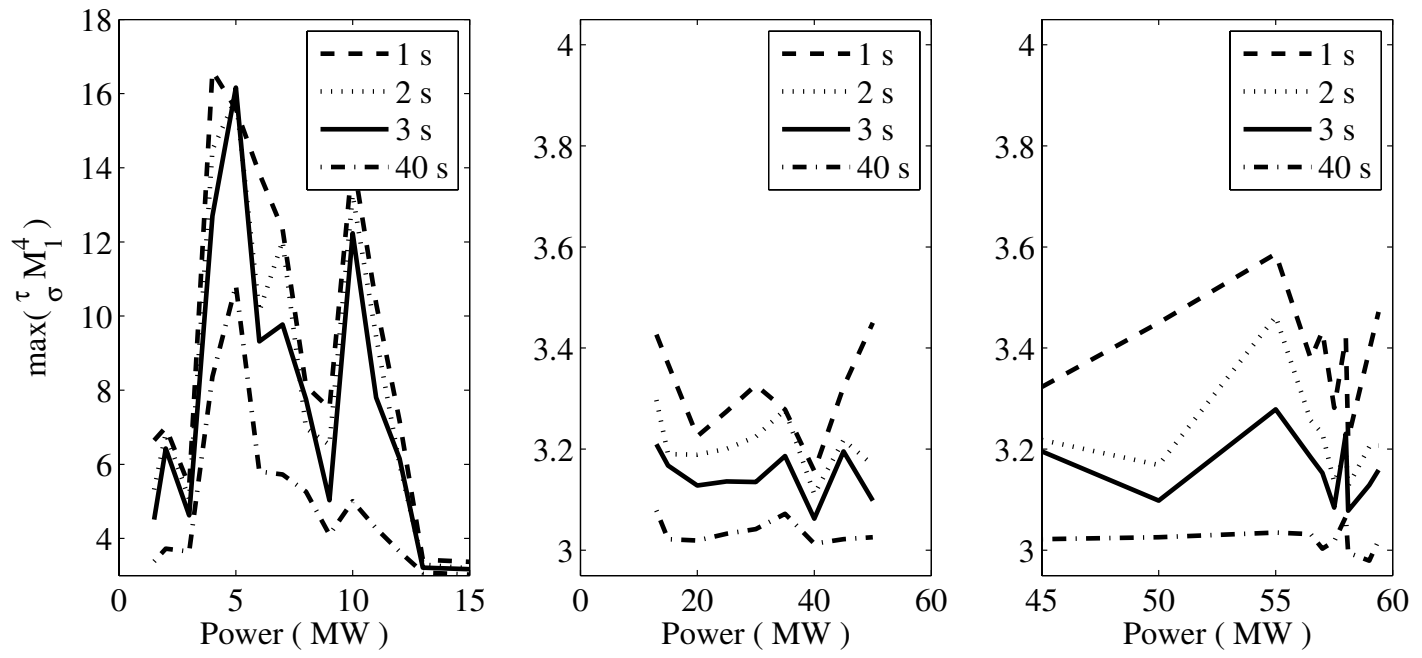

Fig. 5. Maximum moment of the velocity calculated using different sample lengths for three power ranges in the frequency range $100-1000 \mathrm{~Hz}$. The maximum values are obtained from several samples.

tion, short-term cavitation and cavitation-free cases. The same knowledge-based cavitation index $I_{C}^{*}$ shown Figure 2 is used partly for tuning and partly for testing in all the frequency ranges. The groups analysed for the signals $x^{(1)}, x^{(2)}, x^{(3)}$, and $x^{(4)}$ contain rms value and either the generalised moment or peak value of the signal.

The value ranges of the moments are drastically lower in the high power range (Fig. 3(c)) than in the low power range (Fig. 3(a)). This can be handled by means of specific sets of scaling functions, or by means of both rms values and moments. Cavitation in the high power range is less serious than in the low power range.

\section{MONITORING}

Cavitation, short-term cavitation and cavitation-free operating conditions need to be detected in condition monitoring. Reliable indicators could be useful in turbine control when large load variations need to be handled.
Cavitation-free conditions are reliably detected with features obtained in the frequency range $10-1000 \mathrm{~Hz}$. The most difficult part is to find differences between cavitation and short-term cavitation. Although the acceleration features provided good fits with the train and test data, they are not sensitive enough for detecting the operating conditions. The features of the higher derivatives $x^{(3)}$ and $x^{(4)}$ have much better overall performance, especially in wider frequency ranges, $10-3000 \mathrm{~Hz}$ and $10-4000 \mathrm{~Hz}$, the features of the signal $x^{(4)}$ provide better results. [Lahdelma and Juuso, 2007]

The new moments ${ }_{\sigma}^{\tau} M_{\alpha}^{p}$ can be used in the same way as kurtosis in the previous studies [Juuso et al., 2007, Lahdelma et al., 2007]. The moments calculated for higher order derivatives $x^{(3)}$ and $x^{(4)}$ are more sensitive than the ones calculated for velocity. The sensitivity of the moment improves when the order $p$ of the moment increases. The order $p$ is selected to be close to four, and the sample time should be fairly short in the cavitation analysis. 
In this study, the sample time $\tau=3 \mathrm{~s}$ provided the most sensitive results. However, sufficiently long signals are required to produce reliable maximum moments and material for analysing short-term cavitation.

On-line cavitation monitoring is feasible with this approach since the analysis does not need high frequency ranges, and the sample times are very short. The moment ${ }_{\sigma}^{\tau} M_{\alpha}^{p}$ can be analysed first and then combined with the rms values to obtain the cavitation index if the moment value exceeds the threshold value. Data compression is very efficient as the detailed analysis only requires the feature values, i.e. the moment and the rms value, of the appropriate samples. Uncertainty can be handled by presenting the indices as time-varying fuzzy numbers analysed from several samples. The classification limits and thresholds can also be considered fuzzy. Generalised moments can also provide informative features for fault diagnosis in bearings and gear boxes.

\section{CONCLUSIONS}

Features of the velocity $x^{(1)}$, acceleration $x^{(2)}$ and higher derivatives $x^{(3)}$ and $x^{(4)}$ were compared in detecting cavitation. The features of the velocity had a very low correlation with the knowledge-based cavitation index. The new generalised moment provides the first indication of a cavitation risk. Moments obtained from the signals $x^{(4)}$ are also highly informative features to be used in modelbased cavitation indicators. Short sample times and relatively low requirements for the frequency ranges make this approach feasible for on-line analysis and power control. The generalised moment can be defined by the order of derivation, the order of the moment and sample time.

\section{REFERENCES}

S. A. Al-Hashmi, F. Gu, and A. D. Ball. Instantaneous angular speed analysis for cavitation monitoring in centrifugal pump. In Proceedings of the 18th International Congress of Condition Monitoring and Diagnostic Engineering Management (COMADEM 2005)), August 31-September 1, 2005, Cranfield, UK, pages 201-209, Cranfield, UK, 2005. Cranfield University Press.

L. Alfayez, D. Mba, and G. Dyson. The application of acoustic emission for detecting incipient cavitation and the best efficiency point of a $60 \mathrm{~kW}$ centrifugal pump: case study. NDTEE International, 38:354-358, 2005.

A. S. Bahaj and L. E. Myers. Fundamentals applicable to the utilisation of marine current turbines for energy production. Renewable Energy, 28:2205-2211, 2003.

C. E. Brennen. Cavitation and bubble dynamics. Oxford University Press, Oxford, 1995.

M. Cudina. Detection of cavitation phenomenon in a centrifugal pump using audible sound. Mechanical Systems and Signal Processing, 17:1335-1347, 2003.

X. Escaler, E. Egusquiza, M. Farhat, F. Avellan, and M. Coussirat. Detection of cavitation in hydraulic turbines. Mechanical Systems and Signal Processing, 20: 983-1007, 2006.

E. Juuso and S. Lahdelma. Intelligent cavitation indicator for Kaplan water turbines. In U. Kumar, A. Parida, and R. B. K. N. Rao, editors, Proceeding of the 19th International Congress on Condition Monitoring and Diagnostic Engineering Management (COMADEM 2006),
June 12-15, 2006, Luleå, Sweden, pages 849-858. Luleå University Press, Luleå, 2006.

E. Juuso, S. Lahdelma, and P. Vähäoja. Feature extraction for vibration analysis of cavitation in Kaplan water turbines. In Proceedings of the 2nd World Congress on Engineering Asset Management and the 4th International Conference on Condition Monitoring - WCEAMCM 2007, June 11-14, 2007, Harrogate, UK. Coxmoor Publishing, Oxford, UK, 2007.

E. K. Juuso. Fault diagnosis based on linguistic equation framework. In T. Ruokonen, editor, Preprints of IFAC Symposium on Fault Detection, Supervision and Safety for Technical Processes SAFEPROCESS'94, Espoo, June 13 - 16, 1994, Vol. 1., pages 374-379, Helsinki, 1994. Hakapaino.

E. K. Juuso. Fuzzy control in process industry: The linguistic equation approach. In H. B. Verbruggen, H.J. Zimmermann, and R. Babuska, editors, Fuzzy Algorithms for Control, International Series in Intelligent Technologies, pages 243-300. Kluwer, Boston, 1999.

E. K. Juuso. Integration of intelligent systems in development of smart adaptive systems. International Journal of Approximate Reasoning, 35:307-337, 2004.

E. K. Juuso and K. Leiviskä. Adaptive expert systems for metallurgical processes. In S.-L. Jämsä-Jounela and A. J. Niemi, editors, Expert Systems in Mineral and Metal Processing, Proceedings of the IFAC Workshop, Espoo, Finland, August 26-28, 1991, IFAC Workshop Series, 1992, Number 2, pages 119-124, Oxford, UK, 1992. Pergamon.

N. Kishor, R. P. Saini, and S. P. Singh. A review on hydropower plant models and control. Renewable and Sustainablre Energy Reviews, 11:776-796, 2007.

S. Lahdelma. On the higher order derivatives in the laws of motion and theirs application to an active force generator and to condition monitoring, Academic Dissertation. University of Oulu, Research report No. 101, Department of Mechanical Engineering, 1995.

S. Lahdelma. Experiences of using higher derivatives in fault detection. Kunnossapito, 16(10):29-32, 2002.

S. Lahdelma and E. Juuso. Advanced signal processing and fault diagnosis in condition monitoring. Insight, 49 (12):719-725, 2007.

S. Lahdelma, J. Strackeljan, and D. Behr. Combination of higher order derivatives and a fuzzy classifier as a new approach for monitoring rotating machinery. In J. McIntyre and D. Sleeman, editors, Proceedings of the 12th International Congress on Condition Monitoring and Diagnostic Engineering Management (COMADEM 1999), July 6-9, 1999, Sunderland, UK, pages 231-241. Coxmoor Publishing, Oxford, 1999.

S. Lahdelma, P. Vähäoja, and E. Juuso. Detection of cavitation in Kaplan water turbines. In Proceedings of 2007 Arctic Summer Conference on Dynamics, Vibrations and Control, August 6-10, 2007, Ivalo, Finland, pages 80-87. Tampere University Press, Tampere, Finland, 2007.

K. Roussopoulos and P. A. Monkewitz. Measurements of tip vortex characteristics and the effect of an anticavitation lip on a model Kaplan turbine blade. Flow Turbulence and Combustion, 64:119-144, 2000. 